\title{
Redes de relações sociais dos idosos residentes em Ermelino Matarazzo, São Paulo: um estudo epidemiológico
}

\author{
Networks of social relations of elderly residents in Ermelino Matarazzo, São Paulo: an \\ epidemiological study
}

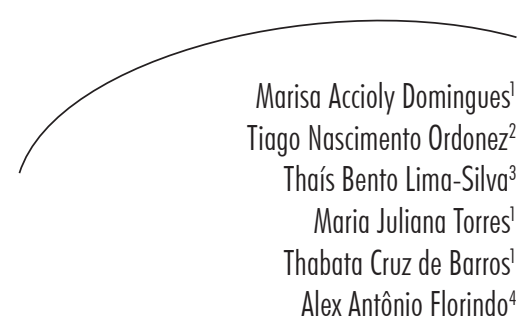

Resumo

Objetivos: Descrever o perfil sociodemográfico e a rede social dos idosos residentes na comunidade de Ermelino Matarazzo, por meio de inquérito epidemiológico. Método: Participaram deste estudo de corte transversal de base domiciliar 382 idosos residentes em Ermelino Matarazzo, com idade igual ou superior a 60 anos. A amostragem foi probabilística, por conglomerados, em dois estágios. Foi realizado sorteio de 35 setores censitários do distrito de Ermelino Matarazzo, na zona leste do município de São Paulo, e, em 2007, realizou-se o recrutamento dos idosos residentes nos domicílios desses setores. Aplicaram-se o questionário sociodemográfico e um instrumento gráfico, o Mapa Mínimo de Relações do Idoso (MMRI). Resultados e Discussão: A análise descritiva dos dados sugere que a população caracterizou-se por idosos de baixa renda e escolaridade, maioria casada ou viúva, aposentada e pensionista. Quanto à rede social, os familiares são o principal suporte dos pesquisados. Quanto ao tamanho da rede, a maioria dos participantes apresentou uma pequena rede social. Quanto ao estado civil, verificou-se que idosos viúvos possuíam uma rede social maior do que a de indivíduos solteiros. As variáveis de idade e renda familiar correlacionaram-se positivamente com o tamanho de rede social. Conclusões: O presente estudo aponta achados que entram em concordância com a literatura, quanto aos membros mais presentes da rede social dos idosos, familiares; ao gênero, feminino; quanto ao tamanho de rede, pequena. Ressaltase que o estudo traz dados representativos da população idosa brasileira por se tratar de estudo com metodologia epidemiológica.

\footnotetext{
Curso de Graduação em Gerontologia, Escola de Artes, Ciências e Humanidades. Universidade de São Paulo. São Paulo, SP, Brasil.

2 Núcleo de Estudo, Pesquisa e Extensão em Gerontologia, Escola de Artes, Ciências e Humanidades. Universidade de São Paulo. São Paulo, SP, Brasil.

3 Programa de Pós-graduação em Neurologia, Faculdade de Medicina. Universidade de São Paulo. São Paulo, SP, Brasil.

4 Curso de Graduação de Ciências da Atividade Física, Escola de Artes, Ciências e Humanidades. Universidade de São Paulo. São Paulo, SP, Brasil.
}

Palavras-chave: Idoso. Rede Social. Percepção de Tamanho. Tamanho de rede. 


\section{Abstract}

Objectives: To describe the sociodemographic and social network of the elderly residents in the community Ermelino Matarazzo, through epidemiological investigation. Method: In this cross-sectional study of 382 elderly residents home base in Ermelino Matarazzo, aged over 60 years. The sample was randomly selected by cluster sampling in two stages. Was randomly selected from 35 census tracts in the district Ermelino Matarazzo, in the east of the city of São Paulo, and in 2007 held the recruitment of older adults living in households in these sectors. We applied the sociodemographic questionnaire and a graphic instrument, the Minimum Map Relations Aging (MMRI). Results and Discussion: A descriptive analysis of the data suggests that the population was characterized by older people with low income and education, most married or widowed, retired and pensioner. As for social networking, family members are the main support of respondents. The size of the network, most participants showed a small social network. Regarding marital status, it was found that elderly widows had a social network larger than that of single individuals. The variables of age and family income were positively correlated with the size of the social network. Conclusions: This study highlights findings that come into agreement with the literature, as more gifts to members of the social network of the elderly, family, gender, female, as the network size, small. It is noteworthy that the study provides data representative of the elderly population because it is a study of epidemiological methodology.
Key words: Elderly. Social Networking. Size Perception. Network Size.

\section{INTRODUÇÃO}

Com o aumento da expectativa de vida, ocorreu um crescimento significativo da população idosa. Para 2020, projeta-se que a expectativa de vida da população seja de 75 anos. ${ }^{1}$ Em relação aos idosos, uma medida interessante é a da expectativa de sobrevida, que em 1996 aumentou 16,3 anos para os homens e 20,4 anos para as mulheres. ${ }^{2} \mathrm{O}$ crescente número de idosos fez com que aumentassem as preocupações e iniciativas governamentais em atender às demandas dessas pessoas, principalmente em ações direcionadas ao suporte social.

Para Neri, ${ }^{3}$ as redes de suporte social são "conjuntos hierarquizados de pessoas que mantêm entre si laços típicos das relações de dar e receber" e são extremamente importantes, já que satisfazem as necessidades sociais dos indivíduos, promovendo e completando os recursos sociais existentes, além de possibilitarem o engajamento social dos idosos.

Almeida et al. ${ }^{4}$ e Assis et al. ${ }^{5}$ garantem que muitos idosos que procuram engajamento social em grupos, como as Universidades Abertas à Terceira Idade, sentem-se contemplados pela oportunidade de aprendizagem e notam a significância dessas redes de suporte social para a saúde e a vida em geral, já que os idosos passam a conviver com a experiência do outro e com a troca de histórias de vida que permitem o crescimento pessoal dos envolvidos.

Além das Universidades Aberta à Terceira Idade, há diversos serviços que visam ampliar a rede de suporte social e se mostram relevantes não apenas nesse contexto, mas também no resgate do prazer, da ludicidade, da melhora na percepção de saúde e felicidade, como afirmam Borini e colaboradores ${ }^{6}$ em seu estudo exploratório realizado com 14 pessoas em um Centro Municipal de Convivência.

Dada a importância das redes de suporte social, compete aos profissionais de saúde envolvidos e comprometidos em prevenir e minimizar os efeitos do envelhecimento desenvolver ações e programas que garantam a ampliação das redes de apoio, contribuindo, assim, para a melhora da qualidade de vida, independência, autonomia e participação da população idosa.? 
Baseando-se nesse contexto, destaca-se que estudos sobre perfil dos idosos e das pessoas que compõem seu suporte social ainda são escassos na literatura. Diante deste quadro, o estudo das redes de Ermelino Matarazzo assume papel importante na identificação do perfil dos idosos e de quais são seus grupos de apoio. A descrição das redes de suporte social é fundamental para a identificação de demandas e com isso a participação da esfera pública por meio de programas de envelhecimento saudável, assistidos por órgãos públicos e que garantam uma qualidade de vida melhor para os idosos e suas redes de suporte. Objetivou-se nesse cenário avaliar o perfil de rede de suporte social de idosos participantes de inquérito domiciliar no bairro de Ermelino Matarazzo, subdistrito da zona leste do município de São Paulo.

\section{MATERIAIS E MÉTODOS}

\section{Participantes}

As variáveis analisadas neste estudo fizeram parte do protocolo da pesquisa "Atividade física e sua relação com variáveis individuais e ambientais na população idosa residente em Ermelino Matarazzo na Zona Leste do município de São Paulo", realizada no ano de 2007. Participaram deste estudo de corte transversal de base domiciliar 383 idosos residentes em Ermelino Matarazzo, com idade igual ou superior a 60 anos. A amostragem foi probabilística, por conglomerados, em dois estágios. Foi realizado sorteio de 35 setores censitários do distrito de Ermelino Matarazzo, na zona leste do município de São Paulo, e a seguir realizou-se o recrutamento dos idosos residentes nos domicílios desses setores. As variáveis analisadas neste estudo fizeram parte do protocolo de pesquisa de um estudo maior. As características da amostra de idosos, cujos dados foram aqui analisados, estão descritas em resultados.

Devido ao fato de o último censo do Instituto Brasileiro de Geografia e Estatística (IBGE) ${ }^{8}$ ter sido realizado oito anos antes da pesquisa, após o sorteio dos 35 setores censitários, foi necessário realizar a contagem de todos os domicílios existentes em cada setor sorteado (arrolamento). Este foi realizado pelos entrevistadores participantes do estudo e se caracterizou pela contagem dos endereços, tendo como referência os mapas e as indicações do IBGE. ${ }^{8}$ Todos os endereços foram registrados, inclusive os imóveis comerciais, igrejas e templos, escolas, imóveis em construção e registro de todos os domicílios de cada setor. Somente as residências ocupadas foram incluídas na coleta e foram procurados idosos em todos os domicílios.

Para sortear os sujeitos no domićlíio, foi utilizada a metodologia de Kish, ${ }^{9}$ que define tabelas aleatórias de acordo com o total de moradores. Cada domicílio recebeu uma tabela definindo qual sujeito deveria ser entrevistado, de acordo com o número de moradores idosos no domicílio. Foram estipuladas oito tabelas diferentes para que os sorteios mantivessem aleatoriedade. Dessa forma, os moradores idosos dos domicílios foram ordenados de acordo com a idade e de maneira decrescente (do mais velho para o mais novo)..$^{10}$

Durante a coleta de dados, primeiramente o entrevistador identificava o domicílio sorteado (com endereço, número estipulado do domić́lio e setor), listava o número total de moradores no domicílio (contando apenas os moradores com 60 anos ou mais) e identificava o sorteado. Após o sorteio, o entrevistador marcava o dia e o horário para a entrevista, que era realizada após o sujeito sorteado assinar o Termo de Consentimento Livre e Esclarecido.

Para participar da amostra deste estudo, os participantes deveriam apresentar idade igual ou superior a 60 anos e residir há pelo menos seis meses no município selecionado. Foram excluídos idosos que apresentaram doenças ou problemas que afetassem as atividades físicas de locomoção ou as atividades físicas de lazer até uma semana antes da abordagem, ou que apresentassem problemas de saúde mental que impedissem o idoso de responder o questionário sozinho. 
Instrumentos

Foi aplicado questionário sociodemográfico, que incluiu idade, renda, anos de escolaridade, estado civil e gênero, para descrever o perfil da amostra recrutada. Para avaliar o suporte social recebido pelo indivíduo, foi aplicado um instrumento gráfico denominado Mapa Mínimo de Relações, que identifica os relacionamentos significativos para o indivíduo, delimitando sua rede de suporte social. ${ }^{11}$ Esse instrumento foi adaptado e modificado por Domingues, ${ }^{12}$ para identificar e caracterizar a rede de suporte social de idosos, sendo submetido a um processo de adequação às demandas dessa população, denominando-se Mapa Mínimo de Relações do Idoso (MMRI).

Sua grande vantagem em relação aos outros tipos de instrumento de avaliação social é o fato de ser um instrumento gráfico de fácil e rápida aplicação. Tal atributo lhe permite identificar e visualizar os vínculos significativos mencionados com presteza. Outra qualidade em relação aos demais instrumentos é que este pode ser aplicado por todos os profissionais de uma equipe multidisciplinar, independentemente de sua formação, desde que capacitado para tanto.

O MMRI é constituído por quatro quadrantes que representam família, amigos, comunidade e relações com os serviços sociais ou de saúde. Sobre esses quadrantes inscrevem-se três áreas, ou seja, um círculo interno de relações mais próximas, cujos contatos ocorrem pelo menos uma vez por semana; um círculo intermediário de relações pessoais com encontros que acontecem pelo menos uma vez por mês; um círculo externo de conhecimento e contatos ocasionais de, no mínimo, uma vez por ano. Os relacionamentos significativos são dispostos nos círculos para simbolizar os diversos graus de proximidade de relacionamento. Para configurar um mapa de relações, é necessário descrever a proximidade do relacionamento segundo a percepção do pesquisado e a frequência com que o contato ocorre. $^{13}$
Os índices de tamanho de rede e de suporte social têm como base a frequência de ações ou registros de possíveis intervenções, caso necessário, recebidas ao longo de um ano. São índices análogos, que se diferenciam quanto ao tipo de auxílio recebido. O Índice de Tamanho da Rede Social (ITRS) leva em conta todos os registros das cinco questões do Mapa Mínimo de Relações do Idoso (MMRI):

1. Quais as pessoas que o(a) visitam pelo menos uma vez por semana, uma vez por mês, uma vez por ano?

2. Com quem o(a) senhor(a) pode contar se desejar ou precisar de alguém para lhe fazer companhia uma vez por semana, uma vez por mês, uma vez por ano?

3. A quem o(a) senhor(a) recorre ou recorreria se precisar de ajuda para cuidar das coisas da casa, como, por exemplo, arrumar, limpar, cozinhar ou fazer compras uma vez por semana, uma vez por mês, uma vez por ano?

4. A quem o(a) senhor(a) recorre ou recorreria se precisar de ajuda para cuidados pessoais, como, por exemplo, trocar de roupa, tomar banho, comer, levantar-se, deitar-se uma vez por semana, uma vez por mês, uma vez por ano?

5. Quem ajuda ou ajudaria o(a) senhor(a) financeiramente se precisar de auxílio para pagar aluguel da sua casa, pagar uma conta, comprar comida, remédio, etc., uma vez por semana, uma vez por mês, uma vez por ano?

Em contrapartida, o Índice de Suporte Social (ISS), compreende apenas as questões 2, 3 e 4, referentes à companhia, auxílio nas atividades domésticas e auxílio para cuidados pessoais, pois estas avaliam melhor o suporte social recebido pelo idoso.

Ambos os índices foram confeccionados a partir da Análise Fatorial Exploratória (Rotação Varimax) dos registros coletados por intermédio do MMRI (total de registros semanal, total de 
registros mensal e total de registros anual), onde se atribuíram pesos diferentes segundo suas frequências. Além disso, extraíram-se da análise supracitada dois fatores:

1. Fator Esporádico: no qual se atribuem pesos maiores aos contatos ou suportes mensais e anuais;

2. Fator Frequente: no qual se atribui peso maior aos contatos ou suporte semanais, em detrimento dos contatos mensais e anuais.

Por fim, com o auxílio da Análise de Agrupamentos (via método $K$-means) e os fatores dos índices instituídos anteriormente, construíram-se os três grupos de indivíduos para cada índice (pequeno, médio e grande). A técnica multivariada consiste em dividir os dados em grupos ou clusters, classificando objetos ou indivíduos em grupos homogêneos, isto é, observando apenas as similaridades ou dissimilaridades entre eles. Desta maneira, obtiveram-se três grupos distintos: pequeno, médio e grande.

\section{Aspectos éticos}

O projeto de pesquisa foi aprovado pelo Comitê de Ética em Pesquisa da Faculdade de Saúde Pública da Universidade de São Paulo, sob o parecer $n^{\circ} 1.488$. Antes de iniciar a avaliação, cada participante da pesquisa assinou um termo de consentimento livre e esclarecido, e, após a conclusão da entrevista, recebeu orientações sobre atividade física e saúde e programas de atividade física desenvolvidos no bairro onde residem.

\section{Análises estatísticas}

As informações obtidas mediante a aplicação dos instrumentos foram submetidas a análise estatística descritiva de natureza univariada e multivariada. Para descrever o perfil da amostra, segundo as diversas variáveis em estudo, foram feitas tabelas de frequência das variáveis categóricas e estatísticas descritivas, como medidas de posição e dispersão, das variáveis contínuas.

Todas as variáveis contínuas de interesse do estudo foram submetidas ao teste de KolmogorovSmirnov e, desse modo, identificou-se a ausência de distribuição normal e que estas exigiriam testes paramétricos. Portanto, para comparação das variáveis contínuas entre dois grupos, foi utilizado o teste U de Mann-Whitney. E entre três grupos ou mais, o teste Kruskall-Wallis, seguido do teste de múltipla comparação de z quando $\mathrm{p}<0,05$.

Para analisar a relação entre as variáveis numéricas, foi utilizado o coeficiente de correlação de Spearman. Valores próximos de +1 indicam forte correlação entre os valores, enquanto que valores próximos de 0 mostram ausência de relação entre as variáveis. ${ }^{14}$

Os dados foram digitados no Programa Epidata versão $3.1 \mathrm{e}$, posteriormente, foram analisados com o programa computacional Statistica 7.0. O nível de significância adotado para os testes estatísticos foi de $5 \%$, ou seja, $\mathrm{p}$-valor $<0,05$

\section{RESULTADOS}

A análise descritiva dos dados sugere que a população caracterizou-se por idosos de baixa renda e escolaridade, maioria casada ou viúva, aposentada e pensionista (ver tabela 1). Quanto à rede de suporte social, os familiares são o principal suporte dos pesquisados. Há fortes evidências de que as filhas são as maiores provedoras de apoio social. Quanto ao tamanho da rede social e de suporte social, a maioria dos participantes apresentou redes de tamanho pequeno, que podem auxiliá-los ao longo do ano com algumas das atividades citadas (visitas, companhia, auxílio para atividades domésticas, auxílio para cuidados pessoais e auxílio financeiro), como mostra a tabela 2. 
Tabela 1 - Distribuição das variáveis sociodemográficas dos participantes, no pré-teste ( $\mathrm{n}=382$ ). São Paulo, SP, 2007.

\begin{tabular}{|c|c|c|c|}
\hline Variáveis & Categorias & $\mathrm{n}$ & $\%$ \\
\hline \multirow[t]{2}{*}{ Sexo } & Masculino & 152 & 39,79 \\
\hline & Feminino & 230 & 60,21 \\
\hline \multirow[t]{6}{*}{ Grupos de idade (em anos) } & 60 a 64 anos & 129 & 33,77 \\
\hline & 65 a 69 anos & 87 & 22,77 \\
\hline & 70 anos ou mais & 166 & 43,46 \\
\hline & Média (DP) & \multicolumn{2}{|c|}{$69,18(7,31)$} \\
\hline & Mediana & \multicolumn{2}{|c|}{68,00} \\
\hline & Mínimo - Máximo & \multicolumn{2}{|c|}{$60,00-97,00$} \\
\hline \multirow[t]{5}{*}{ Estado Civil } & Solteiros & 17 & 4,45 \\
\hline & Casados/União estável & 175 & 45,81 \\
\hline & $\begin{array}{l}\text { Divorciados, Separados, } \\
\text { Desquitados }\end{array}$ & 39 & 10,21 \\
\hline & Viúvos & 150 & 39,27 \\
\hline & Não respondeu & 1 & 0,26 \\
\hline \multirow[t]{7}{*}{ Escolaridade } & Analfabetos & 43 & 11,26 \\
\hline & Ensino Fundamental (incompleto) & 159 & 41,62 \\
\hline & Ensino Fundamental (completo) & 127 & 33,25 \\
\hline & Ensino Médio (incompleto) & 5 & 1,31 \\
\hline & Ensino Médio (completo) & 34 & 8,90 \\
\hline & Ensino Superior (incompleto) & 1 & 0,26 \\
\hline & Ensino Superior (completo) & 13 & 3,40 \\
\hline \multirow[t]{2}{*}{ Aposentadoria } & Não & 126 & 32,98 \\
\hline & $\operatorname{Sim}$ & 256 & 67,02 \\
\hline \multirow[t]{5}{*}{ Renda Familiar } & De 150 a 599 reais & 80 & 20,94 \\
\hline & De 600 a 999 reais & 60 & 15,71 \\
\hline & De 1000 a 1499 reais & 78 & 20,42 \\
\hline & 1500 reais ou mais & 83 & 21,73 \\
\hline & Não responderam & 81 & 21,20 \\
\hline
\end{tabular}


Tabela 2 - Estatísticas descritivas da Rede de Suporte Social dos participantes ( $\mathrm{n}=382$ ). São Paulo, SP, 2007.

\begin{tabular}{|c|c|c|c|c|c|c|c|}
\hline \multirow{2}{*}{ Variáveis } & \multicolumn{7}{|c|}{ Estatísticas Descritivas } \\
\hline & $\mathrm{n}$ & $\%$ & Média & DP & Mínimo & Mediana & Máximo \\
\hline \multicolumn{8}{|l|}{ Rede de Contato Social } \\
\hline Contato esporádico & 382 & 100,00 & 5,01 & 4,50 & 0,00 & 3,82 & 31,87 \\
\hline Contato frequente & 382 & 100,00 & 6,81 & 4,81 & 0,00 & 5,79 & 46,24 \\
\hline Tamanho da Rede de Contato Social & 382 & 100,00 & 1,48 & 0,66 & 1,00 & 1,00 & 3,00 \\
\hline Pequena & 232 & 60,73 & -- & -- & -- & -- & -- \\
\hline Média & 115 & 30,10 & -- & -- & -- & -- & -- \\
\hline Grande & 35 & 9,16 & -- & -- & -- & -- & -- \\
\hline \multicolumn{8}{|l|}{ Rede de Suporte Social } \\
\hline Suporte esporádico & 382 & 100,00 & 2,61 & 3,06 & 0,00 & 1,83 & 28,25 \\
\hline Suporte frequente & 382 & 100,00 & 3,74 & 2,71 & 0,00 & 3,25 & 18,94 \\
\hline Tamanho da Rede Suporte Social & 382 & 100,00 & 1,42 & 0,62 & 1,00 & 1,00 & 3,00 \\
\hline Pequena & 249 & 65,18 & -- & -- & -- & -- & -- \\
\hline Média & 107 & 28,01 & -- & -- & -- & -- & -- \\
\hline Grande & 26 & 6,81 & -- & -- & -- & -- & -- \\
\hline
\end{tabular}

Por meio da tabela 3, quanto ao estado civil, verificou-se que idosos viúvos possuíam uma rede social com contatos frequentes maiores do que a de indivíduos divorciados ou solteiros. Além disso, notou-se que os idosos aposentados, apesar de possuírem contatos esporádicos em nível elevado, possuíam uma rede social maior do que a dos idosos que ainda não estão aposentados (tabela 3).
Documentou-se também que as variáveis de idade e renda familiar correlacionaramse positivamente com os tamanhos de redes sociais e de suporte social, como ilustra a tabela 4, evidenciando que quanto maior a idade e renda, maiores serão as respectivas redes. Concomitantemente, a renda familiar também mostrou-se positivamente associada aos suportes esporádicos e frequentes relacionados ao Índice de Suporte Social. 
Tabela 3 - Pontuação média dos grupos de características sociodemográficas em relação à Rede de Suporte Social (n=382). São Paulo, SP, 2007.

\begin{tabular}{|c|c|c|c|c|c|c|c|c|c|c|c|c|}
\hline \multirow[b]{3}{*}{ Variáveis } & \multicolumn{6}{|c|}{ REDE DE CONTATO SOCIAL } & \multicolumn{6}{|c|}{ REDE DE SUPORTE SOCIAL } \\
\hline & \multicolumn{2}{|c|}{$\begin{array}{c}\text { Contatos } \\
\text { Esporádicos }\end{array}$} & \multicolumn{2}{|c|}{$\begin{array}{l}\text { Contatos } \\
\text { Frequentes }\end{array}$} & \multicolumn{2}{|c|}{ Tamanho } & \multicolumn{2}{|c|}{$\begin{array}{c}\text { Suporte } \\
\text { Esporádico }\end{array}$} & \multicolumn{2}{|c|}{$\begin{array}{l}\text { Suporte } \\
\text { Frequente }\end{array}$} & \multicolumn{2}{|c|}{ Tamanho } \\
\hline & Média & $\pm \mathrm{DP}$ & Média & $\pm \mathrm{DP}$ & Média & $\pm \mathrm{DP}$ & Média & $\pm \mathrm{DP}$ & Média & $\pm \mathrm{DP}$ & Média & $\pm \mathrm{DP}$ \\
\hline \multicolumn{13}{|l|}{ Sexo } \\
\hline Masculino & 4,94 & 3,84 & 6,86 & 4,53 & 1,51 & 0,68 & 2,57 & 2,38 & 3,81 & 2,51 & 1,44 & 0,63 \\
\hline Feminino & 5,06 & 4,89 & 6,77 & 5,00 & 1,47 & 0,64 & 2,64 & 3,45 & 3,70 & 2,84 & 1,40 & 0,61 \\
\hline$p$-valor ${ }^{a}$ & \multicolumn{2}{|c|}{0,497} & \multicolumn{2}{|c|}{0,841} & \multicolumn{2}{|c|}{0,542} & \multicolumn{2}{|c|}{0,287} & \multicolumn{2}{|c|}{0,304} & \multicolumn{2}{|c|}{0,504} \\
\hline \multicolumn{13}{|l|}{ Grupos de idade } \\
\hline 60 a 64 anos & 4,93 & 4,21 & 6,36 & 3,80 & 1,43 & 0,62 & 2,51 & 2,57 & 3,57 & 2,33 & 1,36 & 0,57 \\
\hline 65 a 69 anos & 4,49 & 3,79 & 6,68 & 4,58 & 1,39 & 0,64 & 2,15 & 2,12 & 3,64 & 2,39 & 1,36 & 0,61 \\
\hline 70 anos ou mais & 5,36 & 5,02 & 7,23 & 5,57 & 1,57 & 0,69 & 2,94 & 3,74 & 3,94 & 3,11 & 1,49 & 0,65 \\
\hline$p$-valor ${ }^{\mathrm{b}}$ & \multicolumn{2}{|c|}{0,734} & \multicolumn{2}{|c|}{0,574} & \multicolumn{2}{|c|}{0,053} & \multicolumn{2}{|c|}{0,724} & \multicolumn{2}{|c|}{0,753} & \multicolumn{2}{|c|}{0,122} \\
\hline \multicolumn{13}{|l|}{ Estado Civil } \\
\hline Solteiros & 5,27 & 6,15 & 5,54 & 3,90 & 1,29 & 0,59 & 1,88 & 2,79 & 2,83 & 1,42 & 1,18 & 0,39 \\
\hline Casados/União estável & 4,78 & 3,88 & 6,99 & 4,73 & 1,52 & 0,69 & 2,55 & 2,51 & 3,97 & 2,67 & 1,45 & 0,64 \\
\hline Divorciados/Separados & 4,24 & 3,30 & 4,92 & 2,56 & 1,31 & 0,47 & 2,28 & 2,25 & 2,93 & 1,53 & 1,23 & 0,43 \\
\hline Viúvos & 5,41 & 5,16 & 7,24 & 5,34 & 1,51 & 0,66 & 2,84 & 3,79 & 3,80 & 3,05 & 1,45 & 0,64 \\
\hline$p$-valor ${ }^{b}$ & \multicolumn{2}{|c|}{0,608} & \multicolumn{2}{|c|}{$0,023^{*}$} & 0,2 & & 0,3 & & 0,1 & 70 & 0,1 & \\
\hline Escolaridade (em anos) & & & & & & & & & & & & \\
\hline Ens. Fundamental (até 8 anos) & 5,00 & 4,58 & 6,87 & 4,94 & 1,49 & 0,66 & 2,60 & 3,13 & 3,73 & 2,79 & 1,40 & 0,61 \\
\hline Ens. Médio (de 9 a 11 anos) & 4,89 & 3,99 & 6,19 & 3,89 & 1,36 & 0,63 & 2,81 & 2,75 & 3,53 & 1,71 & 1,38 & 0,54 \\
\hline Ens. Superior (12 anos ou mais) & 5,56 & 4,07 & 7,16 & 4,02 & 1,71 & 0,73 & 2,49 & 2,46 & 4,66 & 3,02 & 1,79 & 0,80 \\
\hline$p$-valor ${ }^{\mathrm{b}}$ & 0,7 & & 0,4 & & 0,1 & & 0,9 & & 0,2 & 55 & 0,1 & \\
\hline Aposentadoria & & & & & & & & & & & & \\
\hline Não & 4,35 & 4,07 & 6,27 & 3,67 & 1,37 & 0,62 & 2,19 & 2,40 & 3,49 & 2,14 & 1,36 & 0,59 \\
\hline $\operatorname{Sim}$ & 5,34 & 4,67 & 7,07 & 5,27 & 1,54 & 0,67 & 2,82 & 3,33 & 3,87 & 2,95 & 1,45 & 0,63 \\
\hline$p$-valor ${ }^{a}$ & 0,0 & & 0,4 & & 0,0 & & 0,1 & & 0,1 & 71 & 0,1 & \\
\hline Renda Familiar & & & & & & & & & & & & \\
\hline De 150 a 599 reais & 4,35 & 4,01 & 6,81 & 5,26 & 1,45 & 0,67 & 2,24 & 2,70 & 3,56 & 3,30 & 1,34 & 0,59 \\
\hline De 600 a 999 reais & 4,87 & 3,70 & 6,55 & 3,96 & 1,50 & 0,62 & 2,36 & 2,31 & 3,54 & 2,68 & 1,32 & 0,57 \\
\hline De 1000 a 1499 reais & 4,73 & 3,99 & 6,46 & 4,01 & 1,40 & 0,63 & 2,57 & 2,77 & 3,46 & 2,00 & 1,35 & 0,58 \\
\hline 1500 reais ou mais & 6,07 & 5,37 & 7,32 & 6,08 & 1,54 & 0,67 & 3,19 & 3,73 & 4,11 & 2,68 & 1,54 & 0,65 \\
\hline$p$-valor ${ }^{\mathrm{b}}$ & 0,1 & & 0,7 & & 0,3 & & 0,1 & & 0,0 & 67 & 0,03 & $9 * *$ \\
\hline
\end{tabular}

Nota: ${ }^{*}$ responderam a questão relacionada à renda $\mathrm{n}=301$.

a. teste U de Mann-Whitney; b. teste Kruskal-Wallis, seguido de teste z de múltipla comparação (Multiple Comparisons z' values) quando $\mathrm{p}<0,05$. *Diferença estatística $(\mathrm{p}<0,05)$ no teste de múltipla comparação entre os estados civis: divorciados/separados<viúvos. **Não há diferença estatística entre os grupos, de acordo com o teste z de múltipla comparação. 
Tabela 4 - Correlação de Spearman entre as variáveis (contínuas) sociodemográficas e de suporte social (n=382). São Paulo, SP, 2007.

\begin{tabular}{|c|c|c|c|c|c|c|}
\hline \multirow[b]{2}{*}{ Variáveis } & \multicolumn{2}{|c|}{ Idade } & \multicolumn{2}{|c|}{ Escolaridade } & \multicolumn{2}{|c|}{ Renda Familiar* } \\
\hline & $\mathrm{r}$ & p-valor & r & p-valor & $\mathrm{r}$ & $p$-valor \\
\hline \multicolumn{7}{|l|}{ Rede de Contato Social } \\
\hline Contato esporádico & 0,04 & 0,439 & 0,00 & 0,979 & 0,12 & 0,044 \\
\hline Contato frequente & 0,08 & 0,098 & $-0,04$ & 0,434 & 0,04 & 0,517 \\
\hline Tamanho da Rede de Contato & 0,11 & 0,032 & $-0,05$ & 0,316 & 0,04 & 0,453 \\
\hline \multicolumn{7}{|l|}{ Rede de Suporte Social } \\
\hline Suporte esporádico & 0,05 & 0,307 & $-0,01$ & 0,828 & 0,12 & 0,030 \\
\hline Suporte frequente & 0,05 & 0,333 & 0,04 & 0,409 & 0,14 & 0,017 \\
\hline Tamanho da Rede de Suporte & 0,11 & 0,038 & 0,04 & 0,469 & 0,14 & 0,016 \\
\hline
\end{tabular}

Nota: $*$ responderam a questão relacionada à renda $n=301$.

\section{DISCUSSÃO}

A predominância de mulheres na velhice é um fato encontrado neste estudo e que corresponde ao perfil sociodemográfico brasileiro, ${ }^{15}$ o que Debert ${ }^{16}$ chama de "feminização da velhice". Estima-se que em 2050 serão seis milhões a mais de mulheres que homens. ${ }^{17} \mathrm{~A}$ longevidade feminina em relação aos homens pode ser considerada um ganho, mas embora este estudo não relate, a literatura aponta que, além de mais longevas, as idosas são mais frágeis, e quanto maior a vulnerabilidade social associada à pobreza, baixa escolaridade e baixo status social, mais estão expostas a situações de violência, abandono e carência de cuidados. ${ }^{18}$

Todas estas questões que apontam para possível vulnerabilidade psicossocial podem ser evidenciadas por meio da utilização de instrumentos de suporte social, tais como o Mapa Mínimo das Relações Sociais dos Idosos. Esses instrumentos permitem identificar o perfil dos idosos, como também a existência ou a falta de grupos de apoio. Esta abordagem faz com que sejam identificadas demandas dentre a população de idosos que devem ser trabalhadas junto às esferas públicas, a partir do desenvolvimento de políticas que suprem as necessidades dos idosos. ${ }^{19}$

Dentre os aspectos de vulnerabilidade encontrados nesta pesquisa, podemos observar que os níveis de baixa escolaridade também foram encontrados em outros estudos que associam baixa escolaridade e baixa renda, menor uso de serviços de saúde e dificuldade de adesão a terapias medicamentosas. ${ }^{20-22}$

Além deste achado, outro dado de interesse encontrado mediante a utilização do instrumento de suporte social diz respeito à renda líquida familiar, já que o Mapa Mínimo das Relações Sociais dos Idosos prioriza também as questões financeiras como um aspecto importante de apoio e suporte social. Com esta informação, foi possível discutir não apenas o simples fato de ter ou não dinheiro suficiente para as necessidades cotidianas, mas também a respeito da mudança da composição familiar quando, muitas vezes, os idosos tornam-se arrimos da família composta por seus filhos, genros ou 
noras e os netos, e as implicações decorrentes dessas novas dinâmicas familiares.

Além dos vínculos familiares, percebe-se a existência de relações de amizade entre os membros da comunidade, não apenas entre vizinhos, mas também em grupos religiosos e em centros de convivência. Essas relações, em sua grande maioria, são significativas ao indivíduo, já que estudos comprovaram que os idosos são mais seletivos em suas amizades e, consequentemente, essas relações de amizades são de extrema importância para questões relacionadas ao suporte social do idoso, uma vez que permitem que estes fiquem mais tempo vivendo na sua comunidade. ${ }^{23,24}$

Como limitação destaca-se que o estudo poderia acompanhar os residentes, para verificar se a rede de suporte social permanecia ou não do mesmo tamanho. Adicionalmente destaca-se que o estudo é descritivo, e que para futuros estudos,

\section{REFERÊNCIAS}

1. Rapizo R. Terapia Sistêmica de Família: da instrução à construção. Rio de Janeiro: Instituto NOOS; 1996.

2. Costa MFFL, Guerra HL, Barreto SM, Guimarães RM. Diagnóstico da situação de saúde da população idosa brasileira: um estudo da mortalidade e das internações hospitalares públicas. Inf Epidemiol do SUS 2000;9(1):43-50.

3. Neri AL. Palavras-chave em gerontologia. 5 a ed. Campinas: Alínea; 2005.

4. Almeida MI, Silva MJ, Araújo MFM. Grupo Vida: adaptação bem sucedida e envelhecimento feliz. RASPP Rev Assoc Saúde Pública de Piauí 1998;1(2):155-62.

5. Assis M, Pacheco LC, Menezes IS. Repercussões de uma experiência de promoção de saúde no envelhecimento: análise preliminar a partir das percepções dos idosos. Textos sobre Envelhecimento 2002;4(7):53-73.

6. Borini MLO, Cintra FA. Representações sociais da participação em atividade de lazer em grupos de terceira idade. Rev Bras Enferm 2002;55(5):568-74.

7. Dias RC, Cardoso RC, Santiago EA, Sampaio AAZ, João AF. Atividades em grupo - alternativa para minimizar os efeitos do envelhecimento. Textos Envelhecimento 2005;8(3):397-410. sugere-se replicação dos métodos e análises de correlações com vaiáveis de saúde.

\section{CONCLUSÕES}

O presente estudo tem caráter epidemiológico e por isso possui grande importância para a Gerontologia e para a esfera pública, uma vez que fornece uma visão mais abrangente das vulnerabilidades sociais populacionais, influenciando diretamente o desenvolvimento de novas políticas públicas voltadas à população idosa.

O estudo traz dados representativos da população idosa brasileira, e aponta achados que estão em concordância com a literatura, quanto aos membros mais presentes da rede de suporte social dos idosos - familiares; ao gênero - feminino; ao tamanho de rede pequena; e quanto ao estado civil, idosos casados apresentavam redes de suporte social maiores.

8. Vieira EB. Manual de Gerontologia: um guia teóricoprático para profissionais, cuidadores e familiares. $2 \mathrm{a}$ ed. Rio de Janeiro: Revinter; 1996.

9. Instituto Brasileiro de Geografia e Estatística, 2000 [acesso em 20 fev 2009]. Disponível em: http://www. ibge.gov.br/home/estatistica/populacao/.

10. Kish L. Survey Sampling. New York: Ed. Wiley; 1965.

11. Salvador EP, Florindo AA, Reis RS, Costa EF. Atividade física de lazer e sua associação com a percepção do ambiente em idosos. Revista de Saúde Pública 2009;43:972-80.

12. Sluzki CE. A rede social na Prática Sistêmica: alternativas terapêuticas. São Paulo: Casa do Psicólogo; 1997. 145 p.

13. Mendes MRSSB, Gusmão JL, Faro ACM, Leite RCBO. A situação social do idoso no Brasil: uma breve consideração. Acta Paul Enferm 2005;18(4):422-6.

14. SESC. Idosos no Brasil: vivências, desafios e expectativas na 3a idade. 2007 [acesso em 1 maio 2011]. Disponível em: http://www2.fpa.org.br/ uploads/1_perfil_sociodemografico_idosos_ brasileiros.pdf .

15. Hair JF, Tatham RL, Anderson RE, Black W. Multivariate Data Analysis. 5a ed. São Paulo: Pearson Education; 2005. 
16. STATISTICA [base de dados na Internet]. Tulsa (EU): Statsoft, Inc. c2004. Data Analysis Software System, version 7 [acesso em 1 maio 2011]. Disponível em: www.statsoft.com

17. Moreira RS, Nico LS, Tomita NE, Ruiz T. A saúde bucal do idoso brasileiro: revisão sistemática sobre o quadro epidemiológico e acesso aos serviços de saúde bucal. Cad. Saúde Pública 2005;21(6):1665-75.

18. Debert GG. A Reinvenção da Velhice: socialização e processo de reprivatização do envelhecimento. São Paulo: Editora da USP/FAPESP; 2004.

19. Brasil: um estudo epidemiológico. Cad. Saúde Pública 2004;20(6):17-37.

20. Domingues MARC, Queiroz Z, Derntl AM. As redes sociais na Senescência. In: Netto MP. Tratado de Gerontologia. São Paulo: Atheneu; 2007. p. 771-781.
21. Domingues MAC, Derntl AM, Ourique SAM. Odontogeriatria: conhecendo o universo social do idoso. Mapa mínimo de relações: adaptação de instrumento gráfico para identificar a rede de suporte social do idoso. JBG J Bras Odonto 2005;1(1):8-18.

22. Feliciano AB, Moraes SA, Freitas ICM. O perfil do idoso de baixa renda no Município de São Carlos, São Paulo, Brasil: um estudo epidemiológico. Cad. Saúde Pública 2004;20(6):1575-85.

23. Barros CA. Grupos de ajuda mútua. In: Zimerman DE, Osório LC. Como trabalhamos com grupos. Porto Alegre: Artmed; 2007. p. 107-17.

24. Lenardt MH. A baixa escolaridade e os medicamentos. Jornal Gazeta do Povo. 2008 [acesso em 1 maio 2011]. Disponível em: http://www. gazetadopovo.com.br/vidaecidadania/conteudo. phtml?tl=1\&id=813572\&tit=A-baixa-escolaridade-eos-medicamentos. 